\title{
Solving Some Derivative Equations Fractional Order Nonlinear Partials Using the Some Blaise Abbo Method
}

\author{
Abdoul wassiha $\mathrm{NEBIE}^{1}$, Frédéric $\mathrm{BERE}^{2}$, Bakari $\mathrm{ABBO}^{3}$, Youssouf PARE ${ }^{1}$ \\ ${ }^{1}$ Département de Mathémétiques, Université Joseph Ki-Zerbo, Burkina Faso \\ ${ }^{2}$ Institut des sciences, Burkina Faso \\ Correspondence: Youssouf PARE, Département de Mathémétiques, Université Joseph Ki-Zerbo, Burkina Faso
}

Received: December 14, 2020 Accepted: March 17, 2021 Online Published: March 28, 2021

doi:10.5539/jmr.v13n2p101 URL: https://doi.org/10.5539/jmr.v13n2p101

\begin{abstract}
In this paper, we propose the solution of some nonlinear partial differential equations of fractional order that modeled diffusion, convection and reaction problems. For the solution of these equations we will use the SBA method which is a method based on the combination of the Adomian Decomposition Method (ADM), the Picard's principle and the method of successive approximations.
\end{abstract}

Keywords: nonlinear time-fractional partial equation, Caputo fractional derivative, Som Blaise Abbo Method (SBA)

\section{Introduction}

The modeling of different phenomena leads in most cases to equations. These equations, mainly functional, are of several types: differential, integral, integro-differential, partial integro-differential and partial derivative (ABBAOUI, K. 1994; ABBAOUI, K. \& CHERRUAULT, Y. 1995; BAKARI, A. \& et al., 2006; MAMPASSI, B. \& et al. 2003) . In recent years, engineers have been opting much more for fractional equations models because the description of systems is more accurate when the fractional derivative is used (KHALOUTA, A. \& KADEM, A, 2019; HAMMOUCH, Z. \& MEKKAOUI, T., 2012; KHALOUTA, A. \& KADEM, A, 2019) . The modeling of systems constited certainly a great progress. However the resolution of different models has created new challenges, which have led researchers to develop several methods of resolution. Among these methods we have analytical methods, discretization methods, asymptotic methods. A particular interest of fractional derivation is related to mechanical modeling of gums and rubbers, in short, all kind of materials that retain the memory of past deformations and whose behaviour is said to be visco-elastic (KHALOUTA, A. \& KADEM, A , 2019; N'GUEREKATA, G. M., 2009; KILBAS, A. A. \& et al., 2006) The Som Blaise-Abbo (SBA) method is a combination of two methods and a principle: Adomian Decomposition Method (ADM), the method of successive approximations and the Picard's principle. The fundamental idea of this method is the construction of a solution at each step in the form of a series that approaches the exact solution of the given problem. The terms of this series are determined using an iterative scheme called algorithm (ABBAOUI, K. 1994; ABBAOUI, K. \& CHERRUAULT, Y. 1995; BAKARI, A. \& et al., 2006; YOUSSOUF, P. \& et al., 2019)

\section{Preliminaries and Definition}

In this section, we present some basic definitions and properties of the fractional calculus theory which are used further in this paper ( KHALOUTA, A. \& KADEM, A , 2019; HAMMOUCH, Z. \& MEKKAOUI, T., 2012; KHALOUTA, A. \& KADEM, A , 2019; N'GUEREKATA, G. M., 2009; KILBAS, A. A. \& et al., 2006) .

Definition 2.1. The Euler Gamma function is defined by:

$$
\Gamma(z)=\int_{0}^{+\infty} t^{z-1} e^{-t} d t
$$

with $z$ is any complex number such that $\operatorname{Re}(z)>0$. The function is strictly decreasing on $] 0 ; 1]$.

Definition 2.2. The Beta function is defined by:

$$
\beta(u, z)=\int_{0}^{1}(1-t)^{u-1} t^{z-1} d t
$$

with $R_{e}(z)>0, R_{e}(u)>0$.

The relation between the beta function and the gamma function is given by: 


$$
\beta(u, z)=\frac{\Gamma(u) \Gamma(z)}{\Gamma(u+z)} .
$$

This relation allows us to conclude that $\beta(u, z)=\beta(z, u)$

Definition 2.3. The Mittag-Leffter function is defined by :

$$
E_{\alpha}(z)=\sum_{k=0}^{+\infty} \frac{z^{k}}{\Gamma(k \alpha+1)}
$$

where $z$ is a complex, $\alpha$ is a strictly positive real.

Definition 2.4. Let $f \in C([a, b])$. The operator $I_{a}^{\alpha}$ defined on $[a ; b]$ by:

$$
\left(I_{a}^{n} h\right)(t)=\frac{1}{\Gamma(\alpha)} \int_{a}^{t}(t-x)^{\alpha-1} h(x) d x
$$

is called Riemann-Liouville fractional integral of order $\alpha>0$.

Propriety 2.1. Let $\alpha$ and $\beta$ two complex numbers, $f \in C([a, b])$.

$$
\begin{aligned}
& \text { i) } I_{a}^{\alpha}\left(I_{a}^{\beta} f\right)=I_{a}^{\alpha+\beta}, \quad R_{e}(\alpha)>0, R_{e}(\beta)>0 \\
& \text { ii) } \frac{d}{d t}\left(I_{a}^{\alpha} f\right)(t)=\left(I_{a}^{\alpha-1} f\right)(t), \quad R_{e}(\alpha)>1 \\
& \text { iii) } \left.\lim _{\alpha \rightarrow 0^{+}} I_{a}^{\alpha} f\right)=f(t), \quad R_{e}(\alpha)>0
\end{aligned}
$$

Definition 2.5. The (left-sided) Caputo-type fractional, derivative of order $\alpha>0$ of a function $f \in C_{-1}^{m}(m=1,2, \ldots$.$) is$ given by:

$$
\left\{\begin{array}{l}
{ }^{c} D^{\alpha}=I^{n-\alpha} u^{(n)}=\frac{\partial^{\alpha} u(x, s)}{\partial s^{\alpha}}=\frac{1}{\Gamma(n-\alpha)} \int_{a}^{t}(t-s)^{n-\alpha-1} \frac{\partial^{n} u(x, s)}{\partial s^{n}} d s, \text { if } \quad n-1<\alpha<n \\
\frac{d^{m}}{d t^{m}} f, \text { if } \quad \alpha=n
\end{array}\right.
$$

where $n=[\alpha]+1$ is the integer part of the real number

\section{Description of SBA Method}

Consider the following functional equation (ABBAOUI, K. 1994; ABBAOUI, K. \& CHERRUAULT, Y. 1995; BAKARI, A. \& et al., 2006; YOUSSOUF, P. \& et al. 2019 ) :

$$
A u=f
$$

where $A: H \rightarrow H$ is either a linear operator or not ; $H$ is a real Hilbert space ; $u \in H$ and $f \in H$.

By setting : $A=L-R-N$, where $L$ is a linear operator assumed to be invertible ; $R$ a linear operator and $N$ a non-linear operator.

The result is :

$$
L u-R u-N u=f
$$

By applying $L^{-1}$, the inverse of $L$ to (7), the following canonical Adomian form is obtained:

$$
u=\theta+L^{-1}(f)+L^{-1}(R(u))+L^{-1}(N(u))
$$

where $\theta$ is such that $L(\theta)=0$.

Applying the method of successive approximations (9) we get :

$$
u^{k}=u^{k}(0)+L^{-1}\left(f^{k}\right)+L^{-1}\left(R\left(u^{k}\right)\right)+L^{-1}\left(N\left(u^{k-1}\right)\right), k \geq 1
$$


From (10) we obtain the following Adomian algorithm, also known as the SBA algorithm:

$$
\left\{\begin{array}{l}
u_{0}^{k}=u^{k}(0)+L^{-1}\left(f^{k}\right)+L^{-1}\left(N\left(u^{k-1}\right)\right), k \geq 1 \\
u_{n}^{k}=L^{-1}\left(R\left(u_{n-1}^{k}\right)\right), n \geq 1
\end{array}\right.
$$

Thus, we combine ideas taken from these two classical techniques in order to derive an approximate schematic.

Then the solution at each step will be calculated by :

$$
u^{k}=\sum_{n=0}^{+\infty} u_{n}^{k}, k \geq 1
$$

The approximate solution $\mathrm{u}$ is given by :

$$
u=\lim _{k \rightarrow+\infty} u^{k}
$$

An important step in the SBA numerical method is the choice of the first iteration term $u^{0}$ of the successive approximation scheme.

Thus, the following principle, called Picard's principle, is proposed.

Picard's principle : choice of $u^{0}$ The principle consists in choosing the first iteration term $u^{0}$ of the approximation scheme in order to obtain algorithms that converge faster to the exact solution. Let's choose $u^{0}$ such that $N\left(u^{0}\right)=0$.

This choice allows in the first iteration to solve only one linear problem and this principle will be checked at each iteration before moving on the calculations.

\section{Convergence :}

Let's consider the following non-linear fractional problem:

$$
\text { (P) }\left\{\begin{array}{l}
{ }^{c} D_{t}^{\alpha} u=L(u)+N(u) \\
u_{0}=f
\end{array}\right.
$$

where $u: H \rightarrow H$ is a known function; $f$ is a continuous function given in the Hilbert space $H$; $L$ is a linear operator and $N$ is a non-linear operator.

\section{Theoreme 3.0.1.}

The SBA algorithm associated with problem $(P)$ is defined for $k \geq 1$ by

$$
\left(P_{S B A}^{k}\right)\left\{\begin{array}{l}
u_{0}^{k}=f+N\left(\tilde{u^{k-1}}\right) \\
u_{n}^{k}=I_{t}^{\alpha}\left(u_{n-1}^{k}\right), n \geq 1
\end{array}\right.
$$

where $I_{t}^{\alpha}()=.\frac{1}{\Gamma(\alpha)} \int_{0}^{t}(t-s)^{\alpha-1}() d$.$s and \tilde{N}\left(u^{k-1}\right)=I_{t}^{\alpha}\left(N\left(u^{k-1}\right)\right)$

Assuming that the following assumptions are verified :

$H_{1}$ : there is $u^{0} \in H$ such that $\tilde{N}\left(u^{0}\right)=0$

$\mathrm{H}_{2}$ : the algorithm:

$$
\left(P^{1}\right)\left\{\begin{array}{l}
u_{0}^{1}=f \\
u_{n}^{1}=I_{t}^{\alpha}\left(u_{n-1}^{1}\right), n \geq 1
\end{array}\right.
$$

converges to the $u^{1}$ solution.

$H_{3}$ : the solution $u^{1} \in H$ is such that $\tilde{N}\left(u^{1}\right)=0 \forall k \geq 2$ the :

$$
\left(P_{S B A}^{k}\right)\left\{\begin{array}{l}
u_{0}^{k}=f, k \geq 2 \\
u_{n}^{k}=I_{t}^{\alpha}\left(u_{n-1}^{k}\right), n \geq 1
\end{array}\right.
$$


converges to the solution $u^{1}=u^{k}, \forall k \geq 2$ and $u=\lim _{k \rightarrow+\infty} u^{k}$ is the only solution to the problem.

\section{Proof:}

- Under hypothesis $H_{1}$, it $u^{0} \in H$ such that $N\left(u^{0}\right)=0$.

- And under the $\mathrm{H}_{2}$ hypothesis the :

$$
\left(P^{1}\right)\left\{\begin{array}{l}
u_{0}^{1}=f \\
u_{n}^{1}=I_{t}^{\alpha}\left(u_{n-1}^{1}\right), n \geq 1
\end{array}\right.
$$

converges to the $u^{1}$ solution.

- Under the assumption $H_{3}$, we have $\tilde{N}\left(u^{1}\right)=0$ and the algorithm :

$$
\left(P_{S B A}^{2}\right)\left\{\begin{array}{l}
u_{0}^{k}=f \\
u_{n}^{2}=I_{t}^{\alpha}\left(u_{n-1}^{2}\right), n \geq 1
\end{array}\right.
$$

is equivalent to the algorithm $\left(P_{S B A}^{1}\right)$ which converges to the solution $u^{1}=u^{2}$.

Thus we have : $\widetilde{N}\left(u^{1}\right)=\widetilde{N}\left(u^{2}\right)=0$

- In step $k=p$, we have $\widetilde{N}\left(u^{p-1}\right)=0$ and the algorithm :

$$
\left(P_{S B A}^{2}\right)\left\{\begin{array}{l}
u_{0}^{k}=f \\
u_{n}^{2}=I_{t}^{\alpha}\left(u_{n-1}^{2}\right), n \geq 1
\end{array}\right.
$$

converges to $u^{p}=u^{1}$, because the algorithms $\left(P_{S B A}^{1}\right),\left(P_{S B A}^{2}\right), \ldots,\left(P_{S B A}^{p}\right)$ are equivalent.

Thus with these hypotheses we have $\widetilde{N}\left(u^{1}\right)=\widetilde{N}\left(u^{2}\right)=\cdots=\widetilde{N}\left(u^{p}\right)=0$

- In step $k=p+1$, we have $\widetilde{N}\left(u^{p}\right)=0$ and the algorithm :

$$
\left(P_{S B A}^{p+1}\right)\left\{\begin{array}{l}
u_{0}^{p+1}=f \\
u_{n}^{p+1}=I_{t}^{\alpha}\left(u_{n-1}^{p+1}\right), n \geq 1
\end{array}\right.
$$

converges to $u^{p+1}=u^{1}$, because the algorithms $\left(P_{S B A}^{1}\right),\left(P_{S B A}^{2}\right), \cdots,\left(P_{S B A}^{p}\right)$ are equivalent.

The different $P_{S B A}^{k}$ for $k \geq 1$ being equivalent, we have: $u^{1}=u^{2}=\cdots=u^{p}$ and $u=\lim _{k \rightarrow+\infty} u^{k}$

\section{Uniqueness of the solution}

Assuming that problem $(P)$ admits two distincts solutions $u$ and $v$, let $w=u-v$ and let's apply the SBA algorithm with the previous assumptions.

In this case we have:

$$
\left(P_{S B A}^{k}\right)\left\{\begin{array}{l}
w_{0}^{k}=0, k \geq 1 \\
w_{n}^{k}=I_{t}^{\alpha}\left(w_{n-1}^{k}\right), n \geq 1
\end{array}\right.
$$

- In step $k=1$, we have the algorithm :

$$
\left(P_{S B A}^{1}\right):\left\{\begin{array}{l}
w_{0}^{1}=0, k \geq 1 \\
w_{1}^{1}=0 \\
w_{2}^{1}=0 \\
\cdots \\
w_{n}^{1}=0
\end{array}\right.
$$

which converges towards $w^{1}=0$.

- In step $k=2$, we have the algorithm :

$$
\left(P_{S B A}^{2}\right):\left\{\begin{array}{l}
w_{0}^{2}=0, k \geq 1 \\
w_{1}^{2}=0 \\
w_{2}^{2}=0 \\
\cdots \\
w_{n}^{2}=0
\end{array}\right.
$$


which converges towards $w^{2}=0$ :

- So the solution to the problem :

$$
\left(P_{S B A}^{k}\right):\left\{\begin{array}{l}
w_{0}^{k}=0, k \geq 1 \\
w_{n}^{k}=I_{t}^{\alpha}\left(w_{n-1}\right), n \geq 1
\end{array}\right.
$$

at each step $\mathrm{k}$ is $w^{k}=0$.

The solution of the problem is given by : $w=\lim _{k \rightarrow+\infty} w^{k}=0$.

This result leads to $u=v$ which is absurd. Hence the uniqueness of the solution

\section{Applications}

\section{Exemple 4.1.}

Let's consider the problem of nonlinear temporal fractionnal convection-reaction given by:

$$
\left\{\begin{array}{l}
{ }^{c} D_{t}^{\alpha} u=\lambda u+u^{m}-\left(x \frac{\partial u}{\partial x}\right)^{2} u^{m-2} \\
u(x, 0)=x
\end{array}\right.
$$

where $0<\alpha \leq 1, x \in \mathbb{R}$ and $t>0 . m$ is integer greater than or equal to 2 .

By setting :

$$
L u={ }^{c} D_{t}^{\alpha} u, \quad L_{t}^{-1} u=I_{t}^{\alpha}, \quad R u=\lambda u, \quad N u=u^{m}-\left(x \frac{\partial u}{\partial x}\right)^{2} u^{m-2}
$$

We obtain :

$$
L_{t} u=R u+N u
$$

By applying $L_{t}^{-1}$ from $L_{t}$ to (25) we get the canonical form of the problem written us :

$$
u(x, t)=u(x, 0)+L_{t}^{-1}(R u(x, t))+L_{t}^{-1}(N u(x, t))
$$

where $L_{t}^{-1}=I_{t}^{\alpha}$ is the fractional integral.

By applying the method of successive approximations to (26) we obtain :

$$
u^{k}(x, t)=u^{k}(x, 0)+L_{t}^{-1}\left(R u^{k}(x, t)\right)+L_{t}^{-1}\left(N u^{k-1}(x, t)\right), k \geq 1
$$

By applying the Adomian algorithm to (27) we get :

$$
\left\{\begin{array}{l}
u_{0}^{k}(x, t)=u^{k}(x, 0)+I_{t}^{\alpha}\left(N u^{k-1}(x, t)\right), k \geq 1 \\
u_{n}^{k}(x, t)=I_{t}^{\alpha}\left(R u_{n-1}^{k}(x, t)\right), n \geq 1
\end{array}\right.
$$

The solution at each step is given by :

$$
u^{k}(x, t)=\sum_{n=0}^{+\infty} u_{n}^{k}(x, t), k=1 ; 2 ; 3 ; \ldots
$$

Let's apply the Picard principle: By choosing $u^{0}=0$ we have : $N u^{0}=0$.

- First step $k=1:$ derivation of $u^{1}(x, t)$ 
we have:

$$
\left\{\begin{array}{l}
u_{0}^{1}(x, t)=\cos x \\
u_{1}^{1}(x, t)=I_{t}^{\alpha}\left(u_{0}^{1}(x, t)\right)=\frac{x \lambda t^{\alpha}}{\Gamma(\alpha+1)} \\
u_{2}^{1}(x, t)=I_{t}^{\alpha}\left(u_{1}^{1}(x, t)\right)=\frac{x \lambda^{2} t^{2 \alpha}}{\Gamma(2 \alpha+1)} \\
u_{3}^{1}(x, t)=I_{t}^{\alpha}\left(u_{2}^{1}(x, t)\right)=\frac{x \lambda^{3} t^{3 \alpha}}{\Gamma(3 \alpha+1)} \\
\cdot \\
u_{n}^{1}(x, t)=I_{t}^{\alpha}\left(u_{n-1}^{1}(x, t)\right)=\frac{x(\lambda)^{n} t^{n \alpha}}{\Gamma(n \alpha+1)}
\end{array}\right.
$$

The solution of the problem in step $k=1$ is :

$$
\begin{aligned}
u(x, t) & =\sum_{n=0}^{+\infty} u_{n}^{1}(x, t) \\
& =\cos x \sum_{n=0}^{+\infty} \frac{(\lambda)^{n} t^{n \alpha}}{\Gamma(n \alpha+1)} \\
& =x E_{\alpha}\left(\lambda t^{\alpha}\right)
\end{aligned}
$$

where $E_{\alpha}\left(\lambda t^{\alpha}\right)$ is the Mittag-Leffter function.

- Second step $k=2$ : derivation of $u^{2}(x, t)$

Let's compute $u^{2}$ using the following algorithm :

$$
\left\{\begin{array}{l}
u_{0}^{2}(x, t)=u^{2}(x, 0)+I_{t}^{\alpha}\left(N u^{1}(x, t)\right) \\
u_{n}^{2}(x, t)=I_{t}^{\alpha}\left(R u_{n-1}^{2}(x, t)\right), n \geq 1
\end{array}\right.
$$

Let's calculate: $N\left(u^{1}(x, t)\right)$

$$
\begin{aligned}
N\left(u^{1}(x, t)\right) & =\left(x E_{\alpha}\left(\lambda t^{\alpha}\right)\right)^{m}-\left(x \frac{\partial\left(x E_{\alpha}\left(\lambda t^{\alpha}\right)\right)}{\partial x}\right)^{2}\left(x E_{\alpha}\left(\lambda t^{\alpha}\right)\right)^{m-2} \\
& =x^{m}\left(E_{\alpha}\left(\lambda t^{\alpha}\right)\right)^{m}-x^{m}\left(E_{\alpha}\left(\lambda t^{\alpha}\right)\right)^{m}=0
\end{aligned}
$$

This leader to :

$$
\left\{\begin{array}{l}
u_{0}^{2}(x, t)=x \\
u_{1}^{2}(x, t)=\frac{x \lambda t^{\alpha}}{\Gamma(\alpha+1)} \\
u_{2}^{2}(x, t)=\frac{x \lambda^{2} t^{2 \alpha}}{\Gamma(2 \alpha+1)} \\
u_{3}^{2}(x, t)=\frac{x \lambda^{3} t^{3 \alpha}}{\Gamma(3 \alpha+1)} \\
\cdot \\
\cdot \\
u_{n}^{2}(x, t)=\frac{x(\lambda)^{n} t^{n \alpha}}{\Gamma(n \alpha+1)}
\end{array}\right.
$$


The solution of the problem in step $k=2$ is :

$$
\begin{aligned}
u^{2}(x, t) & =\sum_{n=0}^{+\infty} u_{n}^{2}(x, t) \\
& =x \sum_{n=0}^{+\infty} \frac{(\lambda)^{n} t^{n \alpha}}{\Gamma(n \alpha+1)} \\
& =x E_{\alpha}\left(\lambda t^{\alpha}\right)
\end{aligned}
$$

where $E_{\alpha}\left(\lambda t^{\alpha}\right)$ is the Mittag-Leffter function.

Therefore $\forall k \geq 3$, by recurrence we can obtain for each step the solution :

$$
u^{k}(x, t)=x E_{\alpha}\left(\lambda t^{\alpha}\right)
$$

Conclusion: The exact solution to the problem is :

$$
u^{k}(x, t)=x E_{\alpha}\left(\lambda t^{\alpha}\right)
$$

The exact solution of the problem for $\alpha=1$ is :

$$
u(x, t)=x E_{1}(\lambda t)=x e^{\lambda t} .
$$

\section{Numerical analysis:}

In this example we analyze the exact solution for $\alpha=1$ and the approximated solution for some values of $\lambda$ and $\alpha$. We obtain an exact solution defined with the help of the Mittag-Leffter function. The last box of the tables represents the error between the exact solution for $\alpha=1$ and the approximate solution of order 5 for $\alpha=1$.

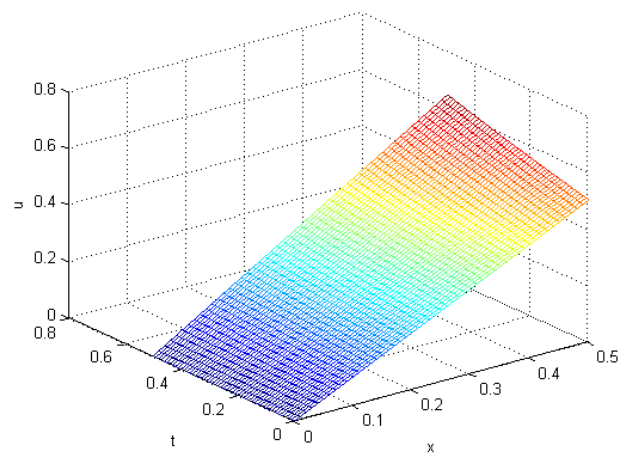

(a) Approximate solution for $\lambda=1, \alpha=0.5$

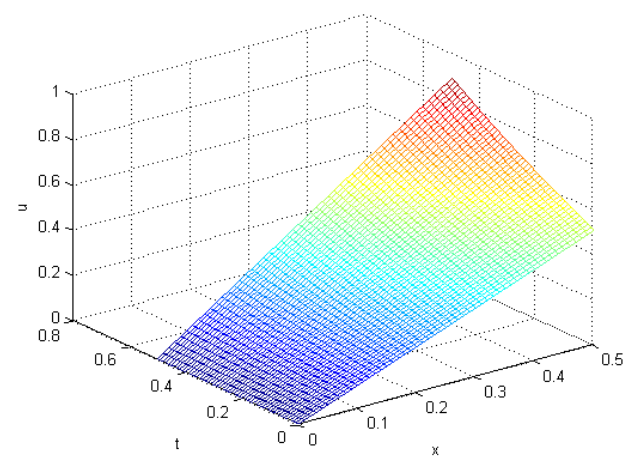

(c) Approximate solution for $\lambda=1, \alpha=0.9$

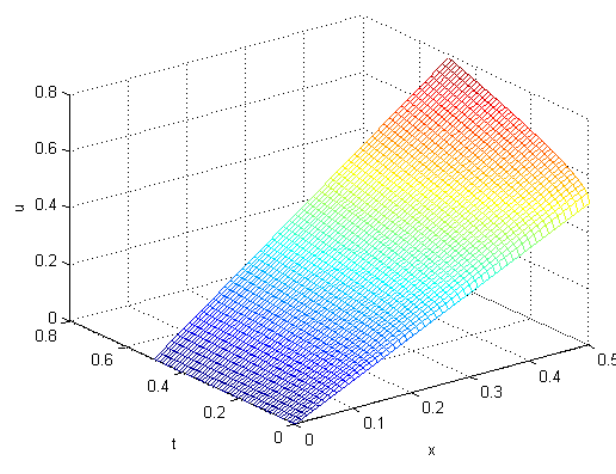

(b) Exacte solution for $\lambda=0.5, \alpha=1$

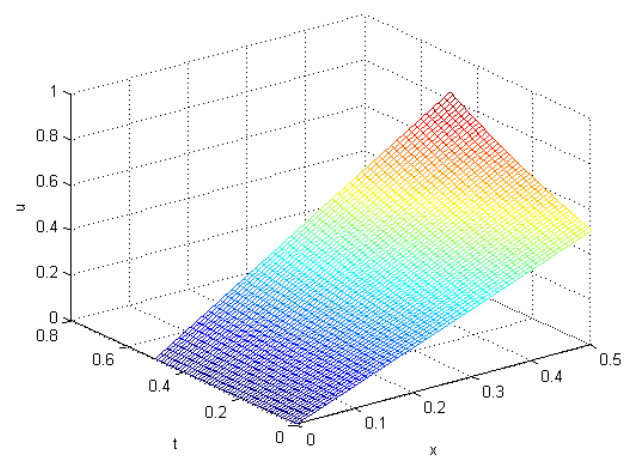

(d) Exacte solution for $\lambda=1, \alpha=1$

Figure 1. Graphs representing the exact solution for $\alpha=1$ and the approximate solution for different values of $\alpha$ and $\lambda$. 
Table 1. Table of numerical values for different values of $\alpha$, with $\lambda=0.03$ and $x=1$

\begin{tabular}{|c|c|c|c|c|c|c|c|}
\hline $\mathrm{t}$ & $\alpha=0.3$ & $\alpha=0.5$ & $\alpha=0.7$ & $\alpha=0.9$ & $\alpha=1$ & $u_{\text {exacte }}$ & $\left|u_{\text {exacte }}-u\right|$ \\
\hline 0.1 & 1.0170 & 1.0108 & 1.0066 & 1.0039 & 1.0030 & 1.0030 & 0 \\
0.2 & 1.0210 & 1.0153 & 1.0108 & 1.0074 & 1.0060 & 1.0060 & 0 \\
0.3 & 1.0238 & 1.0188 & 1.0143 & 1.0106 & 1.0090 & 1.009 & 0 \\
0.4 & 1.0260 & 1.0218 & 1.0176 & 1.0138 & 1.0121 & 1.0121 & 0 \\
0.5 & 1.0278 & 1.0244 & 1.0206 & 1.0169 & 1.0151 & 1.0151 & 0 \\
0.6 & 1.0294 & 1.0268 & 1.0234 & 1.0199 & 1.0182 & 1.0182 & 0 \\
0.7 & 1.0309 & 1.0290 & 1.0262 & 1.0229 & 1.0212 & 1.0212 & 0 \\
\hline
\end{tabular}

Table 2. Table of numerical values for different values of $\alpha$, with $\lambda=1$ et $x=1$

\begin{tabular}{|c|c|c|c|c|c|c|c|}
\hline $\mathrm{t}$ & $\alpha=0.3$ & $\alpha=0.5$ & $\alpha=0.7$ & $\alpha=0.9$ & $\alpha=1$ & $u_{\text {exacte }}$ & $\left|u_{\text {exacte }}-u\right|$ \\
\hline 0.1 & 2.0515 & 1.4866 & 1.2556 & 1.1408 & 1.1052 & 1.1052 & 0 \\
0.2 & 2.5567 & 1.7973 & 1.4595 & 1.2805 & 1.2214 & 1.2214 & 0 \\
0.3 & 3.0094 & 2.1015 & 1.6679 & 1.4305 & 1.3499 & 1.3499 & 0 \\
0.4 & 3.4407 & 2.4144 & 1.8890 & 1.5937 & 1.4918 & 1.4918 & 0 \\
0.5 & 3.8616 & 2.7420 & 2.1268 & 1.7724 & 1.6487 & 1.6487 & 0 \\
0.6 & 4.2775 & 3.0876 & 2.3845 & 1.9685 & 1.8220 & 1.8221 & $10^{-4}$ \\
0.7 & 4.6911 & 3.4530 & 2.6643 & 2.1842 & 2.0136 & 2.0138 & $2.10^{-4}$ \\
\hline
\end{tabular}

\section{Exemple 4.2.}

Let's consider the problem of nonlinear gas-dynamics according to

$$
\left\{\begin{array}{l}
{ }^{c} D_{t}^{\alpha} u=\lambda u-u^{2}-\frac{1}{2} \frac{\partial\left(u^{2}\right)}{\partial x} \\
u(x, 0)=e^{-x}
\end{array}\right.
$$

where $0<\alpha \leq 1, x \in \mathbb{R}$ and $t>0$. By setting :

$$
L u={ }^{c} D_{t}^{\alpha} u, \quad L_{t}^{-1} u=I_{t}^{\alpha}, \quad R u=\lambda u, \quad N u=-u^{2}-\frac{1}{2} \frac{\partial\left(u^{2}\right)}{\partial x}
$$

We obtain :

$$
L_{t} u=R u+N u
$$

By applying $L_{t}^{-1}$ from $L_{t}$ to (40) we get the canonical form of the problem written us :

$$
u(x, t)=u(x, 0)+L_{t}^{-1}(R u(x, t))+L_{t}^{-1}(N u(x, t))
$$

where $L_{t}^{-1}=I_{t}^{\alpha}$ is the fractional integral.

By applying the method of successive approximations to (41) we obtain :

$$
u^{k}(x, t)=u^{k}(x, 0)+L_{t}^{-1}\left(R u^{k}(x, t)\right)+L_{t}^{-1}\left(N u^{k-1}(x, t)\right), k \geq 1
$$

By applying the Adomian algorithm to (42) we get :

$$
\left\{\begin{array}{l}
u_{0}^{k}(x, t)=u^{k}(x, 0)+I_{t}^{\alpha}\left(N u^{k-1}(x, t)\right), k \geq 1 \\
u_{n}^{k}(x, t)=I_{t}^{\alpha}\left(R u_{n-1}^{k}(x, t)\right), n \geq 1
\end{array}\right.
$$


The solution at each step is given by :

$$
u^{k}(x, t)=\sum_{n=0}^{+\infty} u_{n}^{k}(x, t), k=1 ; 2 ; 3 ; \ldots
$$

Let's apply the Picard principle: By choosing $u^{0}=0$ we have : $N u^{0}=0$.

- First step $k=1$ : derivation of $u^{1}(x, t)$

we have:

$$
\left\{\begin{array}{l}
u_{0}^{1}(x, t)=e^{-x} \\
u_{1}^{1}(x, t)=I_{t}^{\alpha}\left(u_{0}^{1}(x, t)\right)=\frac{e^{-x} \lambda t^{\alpha}}{\Gamma(\alpha+1)} \\
u_{2}^{1}(x, t)=I_{t}^{\alpha}\left(u_{1}^{1}(x, t)\right)=\frac{e^{-x} \lambda^{2} t^{2 \alpha}}{\Gamma(2 \alpha+1)} \\
u_{3}^{1}(x, t)=I_{t}^{\alpha}\left(u_{2}^{1}(x, t)\right)=\frac{e^{-x} \lambda^{3} t^{3 \alpha}}{\Gamma(3 \alpha+1)} \\
\cdot \\
\cdot \\
u_{n}^{1}(x, t)=I_{t}^{\alpha}\left(u_{n-1}^{1}(x, t)\right)=\frac{e^{-x}(\lambda)^{n} t^{n \alpha}}{\Gamma(n \alpha+1)}
\end{array}\right.
$$

The solution of the problem in step $k=1$ is:

$$
\begin{aligned}
u(x, t) & =\sum_{n=0}^{+\infty} u_{n}^{1}(x, t) \\
& =e^{-x} \sum_{n=0}^{+\infty} \frac{(\lambda)^{n} t^{n \alpha}}{\Gamma(n \alpha+1)} \\
& =e^{-x} E_{\alpha}\left(\lambda t^{\alpha}\right)
\end{aligned}
$$

where $E_{\alpha}\left(\lambda t^{\alpha}\right)$ is the Mittag-Leffter function.

- Second step $\mathrm{k}=2$ : Calculation of $u^{2}(x, t)$

let's $u^{2}$ using the following algorithm :

$$
\left\{\begin{array}{l}
u_{0}^{2}(x, t)=u^{2}(x, 0)+I_{t}^{\alpha}\left(N u^{1}(x, t)\right) \\
u_{n}^{2}(x, t)=I_{t}^{\alpha}\left(R u_{n-1}^{2}(x, t)\right), n \geq 1
\end{array}\right.
$$

Let's calculate : $\left(N u^{1}(x, t)\right.$

$$
\begin{aligned}
N u^{1}(x, t) & =-\left(e^{-x} E_{\alpha}\left(\lambda t^{\alpha}\right)\right)^{2}-\frac{1}{2} \frac{\partial\left(e^{-x} E_{\alpha}\left(\lambda t^{\alpha}\right)\right)^{2}}{\partial x} \\
& =-e^{-2 x}\left(E_{\alpha}\left(\lambda t^{\alpha}\right)\right)^{2}+e^{-2 x}\left(E_{\alpha}\left(\lambda t^{\alpha}\right)\right)^{2}=0
\end{aligned}
$$


This leader to :

$$
\left\{\begin{array}{l}
u_{0}^{2}(x, t)=e^{-x} \\
u_{1}^{2}(x, t)=\frac{e^{-x} \lambda t^{\alpha}}{\Gamma(\alpha+1)} \\
u_{2}^{2}(x, t)=\frac{e^{-x} \lambda^{2} t^{2 \alpha}}{\Gamma(2 \alpha+1)} \\
u_{3}^{2}(x, t)=\frac{e^{-x} \lambda^{3} t^{3 \alpha}}{\Gamma(3 \alpha+1)} \\
\cdot \\
\cdot \\
u_{n}^{2}(x, t)=\frac{e^{-x}(\lambda)^{n} t^{n \alpha}}{\Gamma(n \alpha+1)}
\end{array}\right.
$$

The solution of the problem in step $k=2$ is :

$$
\begin{aligned}
u^{2}(x, t) & =\sum_{n=0}^{+\infty} u_{n}^{2}(x, t) \\
& =e^{-x} \sum_{n=0}^{+\infty} \frac{(\lambda)^{n} t^{n \alpha}}{\Gamma(n \alpha+1)} \\
& =e^{-x} E_{\alpha}\left(\lambda t^{\alpha}\right)
\end{aligned}
$$

where $E_{\alpha}\left(\lambda t^{\alpha}\right)$ is the Mittag-Leffter function.

Therefore $\forall k \geq 3$, by recurrence we can obtain for each step the solution :

$$
u^{k}(x, t)=e^{-x} E_{\alpha}\left(\lambda t^{\alpha}\right)
$$

Conclusion : The exacte solution of the problem is :

$$
u(x, t)=\lim _{k \rightarrow+\infty} u^{k}(x, t)=e^{-x} E_{\alpha}\left(\lambda t^{\alpha}\right)
$$

The exacte solution of the problem for $\alpha=1$ is :

$$
u(x, t)=e^{-x} E_{1}(\lambda t)=e^{\lambda t-x}
$$

Numerical analysis In this example we analyze the exact solution for $\alpha=1$ and the approximated solution for some values of $\lambda$ and $\alpha$. We obtain an exact solution defined with the help of the Mittag-Leffter function. The last box of the tables represents the error between the exact solution for $\alpha=1$ and the approximate solution of order 5 for $\alpha=1$.

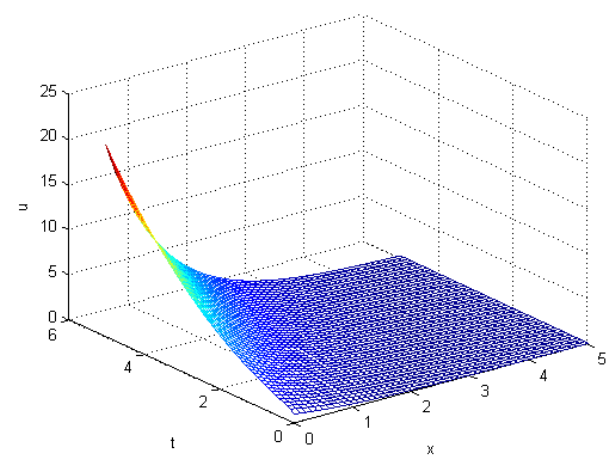

(a) Approximate solution for $\lambda=0.8, \alpha=0.5$

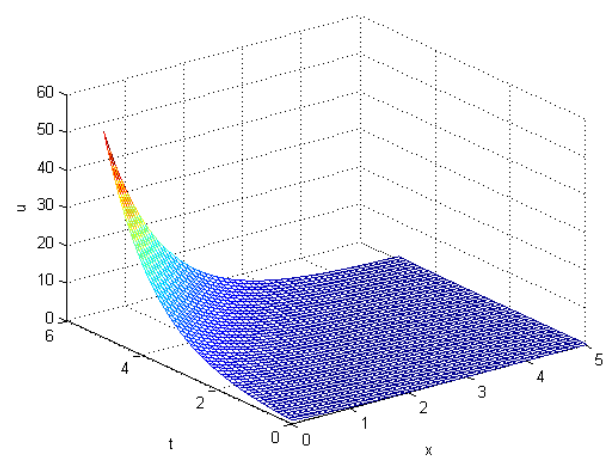

(b) Exacte solution for $\lambda=0.8, \alpha=1$ 


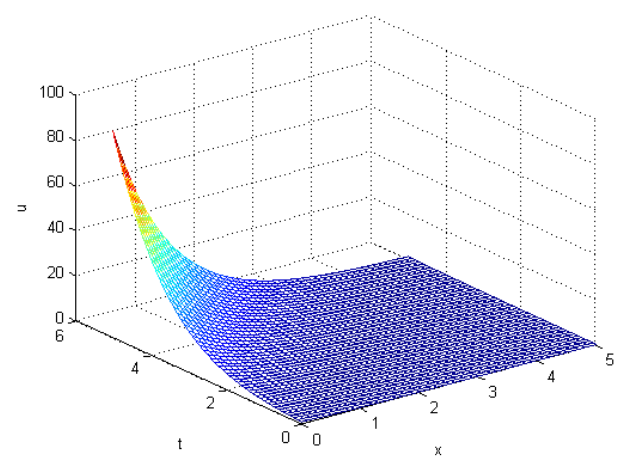

(c) Approximate olution for $\lambda=1, \alpha=1$

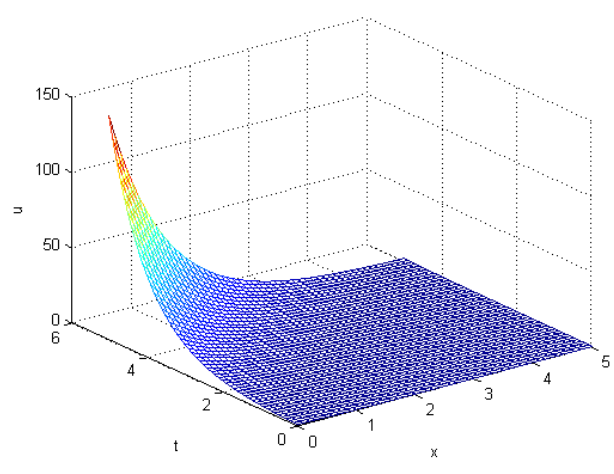

(d) Exacte solution $\lambda=1, \alpha=1$

Figure 2. Graphs representing the exacte solution for $\alpha=1$ et de approximation solution for different values of $\alpha$ and $\lambda$

Table 3. Table of numerical values for different values of $\alpha$, with $\lambda=0.4$ and $x=1$

\begin{tabular}{|c|c|c|c|c|c|c|c|}
\hline $\mathrm{t}$ & $\alpha=0.4$ & $\alpha=0.6$ & $\alpha=0.8$ & $\alpha=0.9$ & $\alpha=1$ & $u_{\text {exacte }}$ & $\left|u_{\text {exacte }}-u\right|$ \\
\hline 0.1 & 0.4692 & 0.4253 & 0.4009 & 0.3928 & 0.3867 & 0.3867 & 0 \\
0.2 & 0.5115 & 0.4600 & 0.4276 & 0.4160 & 0.4066 & 0.4066 & 0 \\
0.3 & 0.5463 & 0.4911 & 0.4536 & 0.4393 & 0.4274 & 0.4274 & 0 \\
0.4 & 0.5775 & 0.5207 & 0.4795 & 0.4633 & 0.4493 & 0.4493 & 0 \\
0.5 & 0.6066 & 0.5496 & 0.5058 & 0.4880 & 0.4724 & 0.4724 & 0 \\
0.6 & 0.6343 & 0.5782 & 0.5327 & 0.5136 & 0.4966 & 0.4966 & 0 \\
0.7 & 0.6610 & 0.6069 & 0.5603 & 0.5402 & 0.5220 & 0.5220 & 0 \\
\hline
\end{tabular}

Table 4. Table of numerical values for different values of $\alpha, \alpha$, with $\lambda=1$ and $x=1$

\begin{tabular}{|c|c|c|c|c|c|c|c|}
\hline $\mathrm{t}$ & $\alpha=0.4$ & $\alpha=0.6$ & $\alpha=0.8$ & $\alpha=0.9$ & $\alpha=1$ & $u_{\text {exacte }}$ & $\left|u_{\text {exacte }}-u\right|$ \\
\hline 0.1 & 0.6249 & 0.4964 & 0.4375 & 0.4197 & 0.4066 & 0.4066 & 0 \\
0.2 & 0.7700 & 0.5882 & 0.4994 & 0.4711 & 0.4493 & 0.4493 & 0 \\
0.3 & 0.9076 & 0.6802 & 0.5641 & 0.5262 & 0.4966 & 0.4966 & 0 \\
0.4 & 1.0451 & 0.7764 & 0.6337 & 0.5863 & 0.5488 & 0.5488 & 0 \\
0.5 & 1.1852 & 0.8789 & 0.7091 & 0.6520 & 0.6065 & 0.6065 & 0 \\
0.6 & 1.3291 & 0.9887 & 0.7914 & 0.7242 & 0.6703 & 0.6703 & 0 \\
0.7 & 1.4775 & 1.1068 & 0.8815 & 0.8035 & 0.7408 & 0.7408 & 0 \\
\hline
\end{tabular}

\section{Exemple 4.3.}

Let's consider the problem of nonlinear diffusion given by:

$$
\left\{\begin{array}{l}
{ }^{c} D_{t}^{\alpha} u=\lambda \frac{\partial^{2} u}{\partial x^{2}}+u^{3}+\left(\frac{\partial^{2} u}{\partial x^{2}}\right)^{2} \\
u(x, 0)=\cos x
\end{array}\right.
$$

where $0<\alpha \leq 1, x \in \mathbb{R}$ and $t>0$ :

By setting :

$$
L u={ }^{c} D_{t}^{\alpha} u, \quad L_{t}^{-1} u=I_{t}^{\alpha}, \quad R u=\lambda \frac{\partial^{2} u}{\partial x^{2}}, \quad N u=u^{3}+\left(\frac{\partial^{2} u}{\partial x^{2}}\right)^{3}
$$


We obtain :

$$
L_{t} u=R u+N u
$$

By applying $L_{t}^{-1}$ from $L_{t}$ to (55) we get the canonical form of the problem written us

$$
u(x, t)=u(x, 0)+L_{t}^{-1}(R u(x, t))+L_{t}^{-1}(N u(x, t))
$$

where $L_{t}^{-1}=I_{t}^{\alpha}$ is the fractional integral.

By applying the method of successive approximations to (56) we obtain :

$$
u^{k}(x, t)=u^{k}(x, 0)+L_{t}^{-1}\left(R u^{k}(x, t)\right)+L_{t}^{-1}\left(N u^{k-1}(x, t)\right), k \geq 1
$$

By applying the Adomian algorithm to (57) we get :

$$
\left\{\begin{array}{l}
u_{0}^{k}(x, t)=u^{k}(x, 0)+I_{t}^{\alpha}\left(N u^{k-1}(x, t)\right), k \geq 1 \\
u_{n}^{k}(x, t)=I_{t}^{\alpha}\left(R u_{n-1}^{k}(x, t)\right), n \geq 1
\end{array}\right.
$$

The solution at each step is given by :

$$
u^{k}(x, t)=\sum_{n=0}^{+\infty} u_{n}^{k}(x, t), k=1 ; 2 ; 3 ; \ldots
$$

Let's apply the Picard principle: By choosing $u^{0}=0$ we have : $N u^{0}=0$.

- First step $k=1$ : derivation of $u^{1}(x, t)$

we have:

$$
\left\{\begin{array}{l}
u_{0}^{1}(x, t)=\cos x \\
u_{1}^{1}(x, t)=I_{t}^{\alpha}\left(u_{0}^{1}(x, t)\right)=\frac{-\cos x \lambda t^{\alpha}}{\Gamma(\alpha+1)} \\
u_{2}^{1}(x, t)=I_{t}^{\alpha}\left(u_{1}^{1}(x, t)\right)=\frac{\cos x \lambda^{2} t^{2 \alpha}}{\Gamma(2 \alpha+1)} \\
u_{3}^{1}(x, t)=I_{t}^{\alpha}\left(u_{2}^{1}(x, t)\right)=\frac{-\cos x \lambda^{3} t^{3 \alpha}}{\Gamma(3 \alpha+1)} \\
\cdot \\
u_{n}^{1}(x, t)=I_{t}^{\alpha}\left(u_{n-1}^{1}(x, t)\right)=\frac{\cos x(-\lambda)^{n} t^{n \alpha}}{\Gamma(n \alpha+1)}
\end{array}\right.
$$

The solution of the problem in step $k=1$ is :

$$
\begin{aligned}
u(x, t) & =\sum_{n=0}^{+\infty} u_{n}^{1}(x, t) \\
& =\cos x \sum_{n=0}^{+\infty} \frac{(-\lambda)^{n} t^{n \alpha}}{\Gamma(n \alpha+1)} \\
& =\cos x E_{\alpha}\left(-\lambda t^{\alpha}\right)
\end{aligned}
$$

where $E_{\alpha}\left(\lambda t^{\alpha}\right)$ is the Mittag-Leffter function.

- Second step $\mathrm{k}=2$ : derivation of $u^{2}(x, t)$

let's $u^{2}$ using the following algorithm :

$$
\left\{\begin{array}{l}
u_{0}^{2}(x, t)=u^{2}(x, 0)+I_{t}^{\alpha}\left(N u^{1}(x, t)\right) \\
u_{n}^{2}(x, t)=I_{t}^{\alpha}\left(R u_{n-1}^{2}(x, t)\right), n \geq 1
\end{array}\right.
$$


Let's calculate : $\left(N u^{1}(x, t)\right.$

$$
\begin{aligned}
N u^{1}(x, t) & =\left(\cos x E_{\alpha}\left(-\lambda t^{\alpha}\right)\right)^{3}+\left(\frac{\partial^{2}\left(\cos x E_{\alpha}\left(-\lambda t^{\alpha}\right)\right)}{\partial x^{2}}\right)^{3} \\
& =\cos ^{3} x\left(E_{\alpha}\left(-\lambda t^{\alpha}\right)\right)^{3}-\cos ^{3} x\left(E_{\alpha}\left(-\lambda t^{\alpha}\right)\right)^{3}=0
\end{aligned}
$$

This leader is:

$$
\left\{\begin{array}{l}
u_{0}^{2}(x, t)=\cos x \\
u_{1}^{2}(x, t)=\frac{-\cos x \lambda t^{\alpha}}{\Gamma(\alpha+1)} \\
u_{2}^{2}(x, t)=\frac{\cos x \lambda^{2} t^{2 \alpha}}{\Gamma(2 \alpha+1)} \\
u_{3}^{2}(x, t)=\frac{-\cos x \lambda^{3} t^{3 \alpha}}{\Gamma(3 \alpha+1)} \\
\cdot \\
u_{n}^{2}(x, t)=\frac{(-\lambda)^{n} t^{n \alpha}}{\Gamma(n \alpha+1)}
\end{array}\right.
$$

The solution of the problem in step $k=2$ is :

$$
\begin{aligned}
u^{2}(x, t) & =\sum_{n=0}^{+\infty} u_{n}^{2}(x, t) \\
& =\cos x \sum_{n=0}^{+\infty} \frac{(-\lambda)^{n} t^{n \alpha}}{\Gamma(n \alpha+1)} \\
& =\cos x E_{\alpha}\left(-\lambda t^{\alpha}\right)
\end{aligned}
$$

where $E_{\alpha}\left(\lambda t^{\alpha}\right)$ is the Mittag-Leffter function.

Therefore $\forall k \geq 3$, by recurrence we can obtain for each step the solution:

$$
u^{k}(x, t)=\cos x E_{\alpha}\left(-\lambda t^{\alpha}\right)
$$

Conclusion : The exacte solution of the problem is :

$$
u(x, t)=\lim _{k \rightarrow+\infty}=\cos x E_{\alpha}\left(-\lambda t^{\alpha}\right)
$$

The exacte solution of the problem for $\alpha=1$ is :

$$
u(x, t)=\cos x E_{1}(-\lambda t)=\cos x e^{-\lambda t} .
$$

Numerical analysis: In this example we analyze the exact solution for $\alpha=1$ and the approximated solution for some values of $\lambda$ and $\alpha$. We obtain an exact solution defined with the help of the Mittag-Leffter function. The last box of the tables represents the error between the exact solution for $\alpha=1$ and the approximate solution of order 5 for $\alpha=1$.

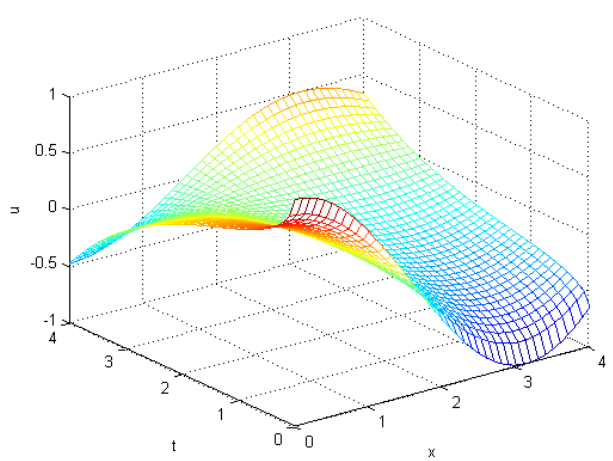

(a) Approximation solution for $\lambda=0.7, \alpha=0.6$

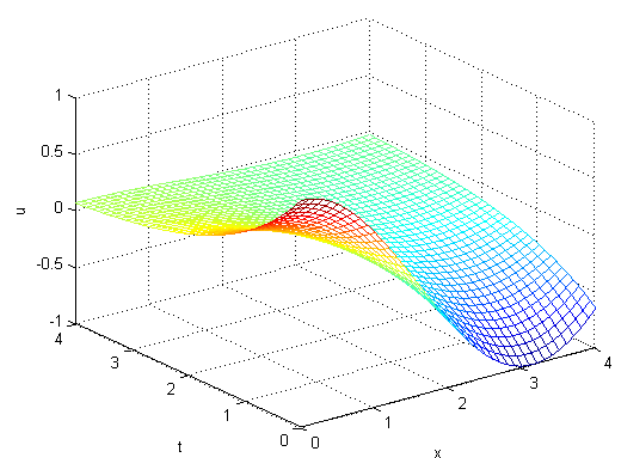

(b) Approximation solution for $\lambda=0.7, \alpha=1$ 


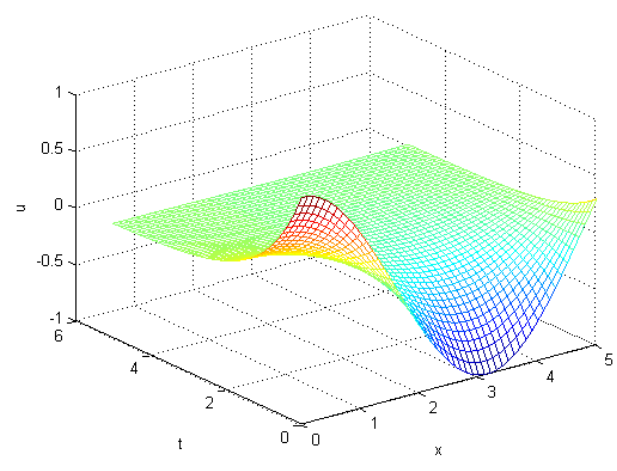

(c) Approximation solution for $\lambda=1, \alpha=1$

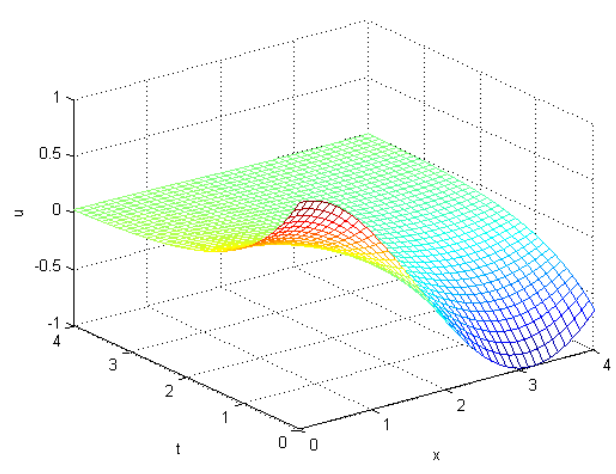

(d) Exacte solution for $\lambda=1, \alpha=1$

Figure 3. Graphs representing the exacte solution for $\alpha=1$ and approximation for different values of $\alpha$ and $\lambda$

Table 5. Table of numerical values for different values of $\alpha$, with $\lambda=0.4$ and $x=1$

\begin{tabular}{|c|c|c|c|c|c|c|c|}
\hline $\mathrm{t}$ & $\alpha=0.3$ & $\alpha=0.5$ & $\alpha=0.8$ & $\alpha=0.9$ & $\alpha=1$ & $u_{\text {exacte }}$ & $\left|u_{\text {exacte }}-u\right|$ \\
\hline 0.1 & 0.4400 & 0.4711 & 0.5050 & 0.5128 & 0.5191 & 0.5191 & 0 \\
0.2 & 0.4216 & 0.4465 & 0.4806 & 0.4903 & 0.4988 & 0.4988 & 0 \\
0.3 & 0.4096 & 0.4289 & 0.4599 & 0.4699 & 0.4792 & 0.4792 & 0 \\
0.4 & 0.4006 & 0.4150 & 0.4416 & 0.4510 & 0.4604 & 0.4604 & 0 \\
0.5 & 0.3933 & 0.4033 & 0.4250 & 0.4335 & 0.4424 & 0.4424 & 0 \\
0.6 & 0.3871 & 0.3932 & 0.4097 & 0.4170 & 0.4250 & 0.4250 & 0 \\
0.7 & 0.3817 & 0.3843 & 0.3956 & 0.4015 & 0.4084 & 0.4084 & 0 \\
\hline
\end{tabular}

Table 6. Table of numerical values for different values of $\alpha$, with $\lambda=1$ and $x=1$

\begin{tabular}{|c|c|c|c|c|c|c|c|}
\hline $\mathrm{t}$ & $\alpha=0.3$ & $\alpha=0.5$ & $\alpha=0.8$ & $\alpha=0.9$ & $\alpha=1$ & $u_{\text {exacte }}$ & $\left|u_{\text {exacte }}-u\right|$ \\
\hline 0.1 & 0.3378 & 0.3909 & 0.4572 & 0.4744 & 0.4889 & 0.4889 & 0 \\
0.2 & 0.3018 & 0.3473 & 0.4056 & 0.4245 & 0.4424 & 0.4424 & 0 \\
0.3 & 0.2732 & 0.3180 & 0.3652 & 0.3826 & 0.4003 & 0.4003 & 0 \\
0.4 & 0.2461 & 0.2948 & 0.3319 & 0.3464 & 0.3622 & 0.3622 & 0 \\
0.5 & 0.2190 & 0.2745 & 0.3036 & 0.3147 & 0.3277 & 0.3277 & 0 \\
0.6 & 0.1909 & 0.2553 & 0.2792 & 0.2868 & 0.2965 & 0.2965 & 0 \\
0.7 & 0.1616 & 0.2361 & 0.2576 & 0.2620 & 0.2682 & 0.2683 & $10^{-4}$ \\
\hline
\end{tabular}

\section{Conclusion}

The SBA numerical method allowed us to solve some partial differential equations of fractional order modeling diffusion, convection and reaction problems such as the gas dynamics equation. It is a very powerful numerical analysis tool for solving this type of problems. This method accelerates the convergence towards the solution and avoids adomial polynomial calculations.

\section{References}

ABBASBANDY, S. (2006). Homotopy perturbation method for quadratic Riccati differential equation and comparison with Adomians decomposition method. Appl. Math. Comput., 172(1), 485-490. https://doi.org/10.1016/j.amc.2005.07.035

ABBAOUI, K., \& CHERRUAULT, Y. (1994). Convergence of the adomian method applied to the nonlinear equations. Math. Comput. Modelling, 20(9), 60-73. https://doi.org/10.1016/0895-7177(94)00163-4 
BAKARI, A., NGARASTA, N., MAMPASSI, B., \& SOME, L. (2006). A new approach of the adomian algoritm for solving nonlinear ordinary or partial differential equations. Far East. J. Math., 23(3), 299-312.

HAMMOUCH, Z., \& MEKKAOUI, T. (2012). Adomian decomposition method for solving a time-fractional BurgerHuxleys equation. Nonlinear Stud., 19, 489C496.

KHALOUTA, A., \& KADEM, A. (2019). A new numerical technique for solving Caputo time-fractional biological population equation. AIMS Mathematics, 4(5), 1307C1319.

KHALOUTA, A., \& KADEM, A. (2019). Comparison of New Iterative Method and Natural Homotopy Perturbation Method for Solving Nonlinear Time-Fractional Wave-Like Equations with Variable Coeficients. Nonlinear Dyn. Syst. Theory, 19, 160-169.

KILBAS, A. A., SRIVASTARA, H. M., \& TRUJILLO, J. J. (2006). Theory and Applications of Fractional Differential Equations. Elsevier, Amsterdam.

MAMPASSI, B., SALEY, B., \& SOME, B. (2003).Solving some Nonlinear Reaction-diffusion equations using the New Adomian Decomposition Method. ADJM., 1(1), 1-9.

N'Gurkata G. M. (2009). A Cauchy problem for some fractional abstract differentiel equation with non local condition. Nonliear Anal., 70, 1873-1876. https://doi.org/10.1016/j.na.2008.02.087

YOUSSOUF, P., YOUSSOUF, M., \& ABDOUL, W. N. (2019). Resolution of nonlinear convection - diffusion - reaction equations of Cauchy's kind by the Laplace SBA method. EJPAM, 12(3), 742-760.

\section{Copyrights}

Copyright for this article is retained by the author(s), with first publication rights granted to the journal.

This is an open-access article distributed under the terms and conditions of the Creative Commons Attribution license (http://creativecommons.org/licenses/by/4.0/). 